\title{
LA PRÁCTICA PROFESIONAL, GESTIÓN Y ANÁLISIS EN LA FACULTAD DE ADMINISTRACIÓN DE LA BENEMÉRITA UNIVERSIDAD AUTÓNOMA DE PUEBLA
}

\author{
THE PROFESSIONAL PRACTICE, MANAGEMENT AND ANALYSIS IN THE \\ FACULTY OF ADMINISTRATION OF THE MERITORIOUS AUTONOMOUS \\ UNIVERSITY OF PUEBLA
}

\begin{abstract}
María Elena Pérez-Terrón ${ }^{1}$ (iD ; Rocío Pérez-y-Terrón ${ }^{2}$ (iD) ; Norma Angélica Santiesteban-López ${ }^{3}$ (iD) ; Dulce María Mora-Valseca ${ }^{4}$.
\end{abstract}

1. Facultad de Administración de la Benemérita Universidad Autónoma de Puebla. pterronm@hotmail.com 2. Facultad de Ciencias Biológicas de la Benemérita Universidad Autónoma de Puebla. rocperez33@hotmail.com

3. Facultad de Administración de la Benemérita Universidad Autónoma de Puebla. asantiesteban2@hotmail.com

4. Facultad de Administración de la Benemérita Universidad Autónoma de Puebla.du.ma.mo.va@hotmail.com

*Correspondencia del Autor: María Elena Pérez-Terrón, correo electrónico: pterronm@hotmail.com.

\section{RESUMEN}

La importancia de esta investigación sobre la Práctica Profesional en las Licenciaturas de Administración Turística y Gastronomía de la Facultad de Administración de la Benemérita Universidad Autónoma de Puebla (BUAP), radica en el objetivo de la información recopilada fue proporcionada por estudiantes de las Licenciaturas, en el desarrollo de sus Prácticas Profesionales (PP) y determinar el grado de aprendizaje del estudiantes en su formación y acompañamiento del docente-tutor, contribuyendo al autoconocimiento interdisciplinario y desarrollo de sus competencias laborales en las empresas de servicios donde realizan las PP. Se diseñó el instrumento de medición y evaluó a 120 estudiantes de ambas Licenciaturas en sus PP en tres periodos de tiempo -cuatrimestres- que son; verano y otoño 2016 y primavera 2017, encuestando 3 apartados: Funciones, Ambiente laboral y Administración con 5 preguntas cada una del instrumento. En base al método científico se consideraron dos tipos de variables; la variable independiente corresponde a la relación existente entre las PP de los estudiantes. La variable dependiente referida a la mejora del aprovechamiento escolar, medible por una entrevista dirigida a los docentes- tutores. Los resultados muestran puntos de coincidencia relevantes que contribuyen a potenciar la gestión de su propio conocimiento en el ámbito laboral.

Palabras Clave: Docentes-Tutores; Educación Superior; estudiantes; práctica profesional.

Cómo citar:

Pérez-Terrón, María Elena; Pérez-y-Terrón, Rocío; Santiesteban-López, Norma Angélica; Mora-Valseca, Dulce María. (2021). La práctica profesional, gestión y análisis en la facultad de administración de la Benemérita Universidad Autónoma de Puebla. Revista de Investigaciones Universidad del Quindío, 33(1), 37-43. https://doi.org/10.33975/riuq.vol33n1.459 


\begin{abstract}
The importance of this research on Professional Practice in Touristic Administration Degrees and Gastronomy of the Faculty of Administration of the Benemerita Universidad Autonoma de Puebla (BUAP), lies in the purpose of the information collected was provided by undergraduate students, in the development of their Professional Practices (PP) and to determine the degree of learning of the students in their training and accompaniment of the tutor teacher, contributing to interdisciplinary self-knowledge and development of their work skills in the service companies where they perform PP. The measuring instrument was designed and evaluated 120 students of both Bachelor's degrees in their PP in three time periods - quarters - that are; summer and autumn 2016 and spring 2017, surveying 3 sections: Functions, Work Environment and Administration with 5 questions each of the instrument. Based on the scientific method, two types of variables were considered; the independent variable corresponds to the relationship between the PP of the students. The dependent variable related to improving school use, measurable by an interview aimed at tutor-teachers. The results show relevant points of coincidence that help to enhance the management of their own knowledge in the field of work.
\end{abstract}

Keywords: Tutor Teachers; Higher Education; students; professional practice.

\section{INTRODUCCIÓN}

En México, la educación superior debe ser uno de los motores de desarrollo social que impulse el conocimiento y la innovación, en una sociedad que tiene imperiosa necesidad de profesionistas con una alta capacitación para enfrentar los retos de la realidad actual, la alta complejidad intelectual, social y organizativa representan las nuevas exigencias que se requieren para la adaptación a los cambios constantes necesarios en un entorno laboral, por ello la importancia de ofrecer al estudiante universitario una forma de aprendizaje integral, la formación de la persona (estudiante) debe incluir el desarrollo del pensamiento crítico, la capacidad de tomar decisiones con apego a lo aprendido y a su propio criterio, las nuevas exigencias provocan cambios en los escenarios laborales. Es así que la BUAP atiende los retos de la demanda laboral, formando profesionistas capaces de transformar su entorno y adaptarse a las nuevas situaciones laborales.

La Benemérita Universidad Autónoma de Puebla es altamente reconocida entre las instituciones, tanto públicas como privadas dentro del sistema de educación superior, como una de la más alta calidad en el estado a nivel nacional e internacional, compitiendo en igualdad de circunstancias con universidades del extranjero, su prestigio se debe a que cuenta con tareas y trabajos de investigación en la formación de profesionales, así como de la ciencia y la cultura, los estudiantes deben de contar con una alta calidad de la enseñanza, para satisfacer las necesidades laborales de los empleadores.

El proceso tutorial es uno de los factores principales para el análisis, gestión y aplicación en la educación superior con el objetivo de fortalecer el trabajo docente hacia el aprendizaje estudiantil, para conocer sus necesidades y proponer soluciones a los problemas de la educación universitaria (Martínez, Pérez, \& Martínez, 2018).

No solo se debe justificar la tutoría como una necesidad en la educación superior desde el punto de vista del estudiante, sino también desde la óptica del docente y de la propia universidad, en este proceso de retroalimentación se tiene una mejor perspectiva de la realidad en el proceso 
enseñanza-aprendizaje (Álvarez González \& Álvarez Justel , 2015).

La Educación Universitaria en el mundo y en este siglo XXI se encuentra en un proceso de universalización y globalización, características del modo de producción económico actual, lo que significa generar modelos innovadores, centrados en el estudiante, la utilización de las tecnologías de la información (TIC), fomentar la autogestión, la formación integral y el aprendizaje a lo largo de la vida y la experiencia de cada comunidad Universitaria. El Modelo Universitario Minerva (MUM) de la BUAP se propone atender con responsabilidad y compromiso las demandas de desarrollo equitativo, sustentable e intercultural de los diversos sectores sociales. Este modelo educativo-académico es resultado de un largo proceso participativo de reflexión y análisis críticos de la comunidad universitaria (BUAP, 2009). Al implementar el MUM en la universidad, la Práctica Profesional es fundamental en la formación curricular de los estudiantes. Las PP constituyen un aspecto medular que todas las universidades deben tomar en cuenta, para el desarrollo profesional de sus estudiantes, de igual manera, el servicio social, lo cual les permitirá saber cuáles son las empresas que realmente apoyan estas actividades. Lo anterior es uno de los objetivos, proporcionar al estudiante la experiencia que contribuya a potenciar la gestión de su propio conocimiento en un ámbito laboral (Gaceta Universidad BUAP, 2013).

\section{METODOLOGÍA}

La investigación se basó en un diseño mixto que integra lo cuantitativo en la medición de las respuestas que dan los estudiantes y lo cualitativo en las respuestas de las entrevistas de los tutores y en la bitácora de lo observado durante el desarrollo de las prácticas profesionales y su experiencia en la incursión laboral.

\section{Instrumento}

Se realizaron dos tipos de enfoque: enfoque cuantitativo y enfoque cualitativo, con el objeto de obtener resultados de problemas propuestos y producir conocimiento científico.

El primer enfoque utilizó la recolección de datos (cuestionario) para comprobar una hipótesis con base en la medición numérica y el análisis estadístico, todo esto para establecer patrones generales de comportamiento y probar teorías formuladas.

\section{Participantes}

Población: Estudiantes de la Facultad de Administración de las Licenciaturas en Administración Turística y Gastronomía correspondiente a la generación 2012.

Muestra: 307 Estudiantes que han realizado la práctica profesional en Administración Turística y 83 en Gastronomía. Con un error máximo aceptable del 5\% y un nivel de confianza del 95\%.

\section{Fórmulas}

$$
\begin{array}{cc}
\text { Fórmula 1 } & \text { Fórmula 2 } \\
\boldsymbol{n}^{\prime}=\frac{\boldsymbol{s}^{2}}{V^{2}} & \boldsymbol{n}=\frac{\boldsymbol{n}^{\prime}}{1+\frac{\boldsymbol{n}^{\prime}}{N}}
\end{array}
$$

Aplicandolas fórmulasseobtuvieronlossiguientes datos: de la muestra de administración turística se determinaron 75 estudiantes encuestados y 45 correspondieron a Gastronomía, siendo la población total encuestada 120 estudiantes (Hernández-Sampieri, 2020).

Los instrumentos de recolección de datos fueron obtenidos por medio de un cuestionario con 15 ítems, que abarcan las dimensiones de funciones, ámbito laboral y administración de la PP, así como un cuestionario dirigido a los docentestutores el cual consistió en 10 preguntas para identificar la experiencia real que tuvieron al ser asignados a esta materia. La concentración de datos se efectuó en hoja de Excel, de acuerdo al formato del diseño de la encuesta realizada a los estudiantes, que se midió en escala de Likert con 15 ítems y tres posibilidades de respuesta que fueron; Totalmente de Acuerdo (T/A), 
$\mathrm{Ni}$ de Acuerdo Ni en Desacuerdo (NA/ND) y Totalmente en Desacuerdo (T/D), obteniendo 1,125 respuestas de la administración turística y 675 para gastronomía. El tratamiento de datos fue realizado de acuerdo a la variable independiente con tres indicadores que son; Funciones, ambiente laboral y administración, así como la variable dependiente con su indicador docentes, aplicadas en ambas licenciaturas, como se muestra en la tabla 1 .

Tabla 1. Tratamiento de datos

\begin{tabular}{|c|c|}
\hline VARIABLES & INDICADORES \\
\hline \multirow{3}{*}{ Independiente } & Funciones \\
\cline { 2 - 2 } & Ambiente laboral \\
\cline { 2 - 2 } & Administración \\
\hline Dependiente & Docentes \\
\hline
\end{tabular}

Fuente: Elaboración propia

\section{RESULTADOS}

Los Resultados que se presentan en las tablas 2,3, y 4 de la variable independiente en el indicador de funciones, ambiente laboral y administración son las que representan las respuestas en porcentajes más altos en cada una de las licenciaturas, las preguntas del indicador funciones corresponden a; 7.- ¿La realización de las actividades desarrolladas en el área fue de acorde a los conocimientos académicos adquiridos en la licenciatura?, 8.- ¿La dependencia otorgó un curso de capacitación al momento de ingresar a realizar las PP?, 9.- ¿Las actividades realizadas en el ámbito laboral cumplieron con sus expectativas?, 10.- ¿Considera adecuado el espacio asignado para la realización de las actividades laborales de la PP?, 11.- ¿Considera adecuadas las herramientas de trabajo para la realización de las actividades encomendadas en la PP?, 12.-¿Su formación académica aumentó la posibilidad de obtener un trabajo en el área que realizó su PP?, y 13.- ¿Considera necesario el uso de una segunda lengua al realizar sus PP?, Dicha información se muestra en la tabla 2 con los porcentajes obtenidos en ambas licenciaturas, en las respuestas correspondientes a totalmente de acuerdo.
Tabla 2. Respuesta indicador de funciones

\begin{tabular}{|c|c|c|}
\hline \multicolumn{3}{|c|}{$\begin{array}{c}\text { OPCIONES DE RESPUESTA - INDICADORES: } \\
\text { FUNCIONES }\end{array}$} \\
\hline \multirow{2}{*}{ Preguntas } & \multicolumn{2}{|c|}{ Totalmente de acuerdo } \\
\cline { 2 - 3 } & LAT & LGAM \\
\hline 7 & 39 & 56 \\
\hline 8 & 46 & 47 \\
\hline 9 & 39 & 56 \\
\hline 12 & 44 & 38 \\
\hline 13 & 60 & 53 \\
\hline \multicolumn{2}{|c|}{ Fuente: Elaboración propia } \\
\hline
\end{tabular}

Con los resultados obtenidos se procedió a graficar el indicador funciones comparando las respuestas por licenciatura en la gráfica 1 .

Gráfica 1. Comparación de resultados de Funciones.

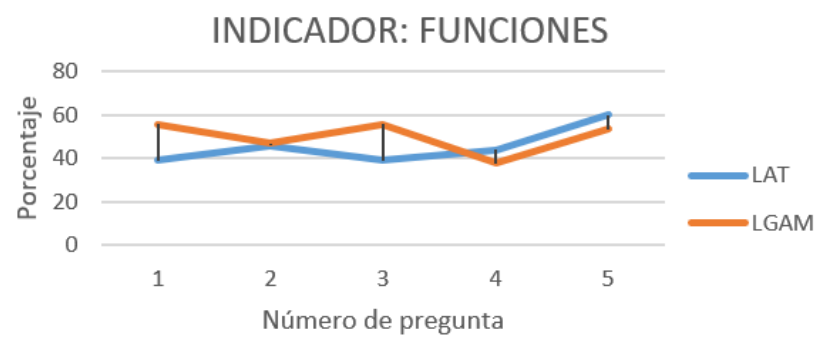

Fuente: Elaboración propia

Como se puede visualizar en la gráfica, la tendencia en las respuestas 7 y 9 favorecen a gastronomía, pues la mayor parte de los estudiantes están totalmente de acuerdo de que los conocimientos adquiridos en su formación los aplican en sus PP, caso contrario de los de turismo los cuales desempeñan funciones distintas a las adquiridas en su formación académica y referente a las respuestas 12 y 13 la tendencia es a favor de los estudiantes de turismo, pues consideran que teniendo el dominio de otro idioma y el desarrollo de sus conocimientos aumenta la posibilidad de inserción laboral una vez concluidas sus PP.

En referencia a la tabla 3 del indicador ambiente laboral con las preguntas; 6.- ¿El proceso de inscripción de la PP es dinámico y explícito? 10.¿Considera adecuado el espacio asignado para la realización de las actividades laborales de la PP? 11.- ¿Considera adecuadas las herramientas de trabajo para la realización de las actividades 
encomendadas en la PP? 14.- ¿La realización de PP brindó motivación y seguridad en el ambiente laboral? Y 15.- ¿Considera que el ambiente laboral fue el idóneo para el desempeño de sus funciones?, los porcentajes obtenidos de las licenciaturas de turismo y gastronomía se muestran en la tabla 3 del indicador ambiente laboral.

Tabla 3. Respuesta indicador de ambiente laboral OPCIONES DE RESPUESTA - INDICADORES:
AMBIENTE LABORAL

\begin{tabular}{|c|c|c|}
\hline \multirow{2}{*}{ Preguntas } & \multicolumn{2}{|c|}{ Totalmente de acuerdo } \\
\cline { 2 - 3 } & LAT & LGAM \\
\hline 6 & 33 & 27 \\
\hline 10 & 47 & 51 \\
\hline 11 & 41 & 53 \\
\hline 14 & 38 & 51 \\
\hline 15 & 52 & 56 \\
\hline \multicolumn{2}{|c|}{ Fuente: Elaboración propia } \\
\hline
\end{tabular}

A continuación, se muestra la gráfica 2.

Gráfica 2. Comparación de resultados de Ambiente laboral.

INDICADOR: AMBIENTE LABORAL

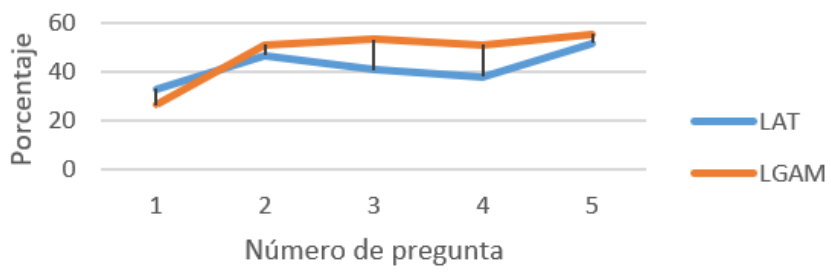

Fuente: Elaboración propia

Los estudiantes de gastronomía realizan sus PP en un ambiente laboral adecuado, contando con todas las herramientas necesarias para llevar a cabo las actividades asignadas, en espacios idóneos y con todas las medidas de seguridad, teniendo los estudiantes la desventaja que, al realizar la gestión en su proceso de asignación, el cupo es limitado por parte de las empresas enlistadas para las PP.

La tabla 4 respecto del indicador administración contiene las siguientes preguntas; 1.- ¿La coordinación de la Práctica Profesional (PP) crítica cumplió adecuadamente con el proceso de elección, para la realización de PP? 2.- ¿La elección de la dependencia o lugar donde realizó la PP fue el deseado? 3.- ¿La documentación solicitada es la adecuada para la inscripción de la PP? 4.- ¿El docente tutor cumplió con el curso de inducción para la realización de la PP? 5.- ¿El horario que eligió para la realización de las PP, fue el adecuado?, las respuestas a las preguntas del indicador administración en la tabla 4 se presentan.

Tabla 4. Respuesta de indicador administración

\begin{tabular}{|c|c|c|}
\hline \multicolumn{3}{|c|}{$\begin{array}{c}\text { OPCIONES DE RESPUESTA - INDICADORES: } \\
\text { ADMINISTRACIÓN }\end{array}$} \\
\hline \multirow{2}{*}{ Preguntas } & \multicolumn{2}{|c|}{ Totalmente de acuerdo } \\
\cline { 2 - 3 } & LAT & LGAM \\
\hline 1 & 65 & 58 \\
\hline 2 & 51 & 60 \\
\hline 3 & 66 & 44 \\
\hline 4 & 49 & 38 \\
\hline 5 & 68 & 69 \\
\hline \multicolumn{3}{|c|}{ Fuente: Elaboración propia } \\
\hline
\end{tabular}

El indicador administración lo podemos en gráfica 3 .

Gráfica 3. Comparación de resultados de Ambiente laboral.

INDICADOR: ADMINISTRACIÓN

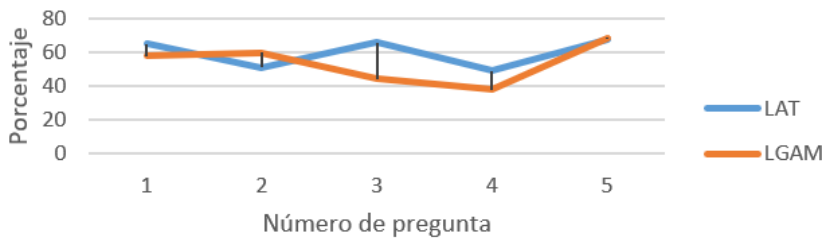

Fuente: Elaboración propia

Los porcentajes más altos obtenidos corresponden a la licenciatura de administración turística respecto de las respuestas a las preguntas 1, 2, 3 , y 4 del indicador administración, pues en el proceso de enseñanza-aprendizaje el programa de estudios está diseñado por áreas de conocimiento económico-administrativas.

La Facultad de Administración de la BUAP cuenta con una Coordinación de Práctica 
Profesional dependiente de la Secretaría Académica, cuyo objetivo es establecer y aplicar las bases del proceso administrativo de acuerdo a los lineamientos establecidos para la realización de la PP, provee información para realizar los trámites y la gestión de convenios e informar y orientar de manera clara, explícita y sencilla a las áreas involucradas en temas de asignación, realización, término y certificación de la Práctica Profesional, de igual manera pretende estandarizar los procesos de entrega y recepción de documentación en este ámbito. $\mathrm{La}$ asignación de los docentes-tutores de las PP son propuestos por la Coordinación de la Facultad y aprobados por las Secretaría Académica. Son ellos los encargados de orientar, guiar y vigilar a los estudiantes que realizan la PP, desde el principio hasta la conclusión, estableciendo los vínculos formales con los empleadores, así como con la Coordinación de la práctica profesional, por último, los tutores califican la aprobación o no de los estudiantes.

\section{CONCLUSIONES}

La recolección de datos a través de cuestionarios aplicados a estudiantes y entrevistas a docentestutores, dan la oportunidad de analizar e interpretar estadísticamente las respuestas, ampliando el panorama de manera global y teniendo una perspectiva particular de cada una de las preguntas para conocer a detalle los aspectos más relevantes que conlleva la PP en el ámbito laboral.
El acompañamiento del tutor hacia el estudiante debe ser continuo para tener la oportunidad de identificar dificultades, necesidades de apoyo y solución de problemas. La PP por su importancia actual como factor de refuerzo de la enseñanzaaprendizaje de los estudiantes en las aulas y como medio para potenciar en la práctica los conocimientos adquiridos, es el primer contacto laboral para poner en acción las habilidades, destrezas y todo el potencial humano de los estudiantes, incluidos la interacción social, el trabajo en equipo, el sentido de responsabilidad y la consecución de metas. Los estudiantes no deben considerar la realización de las PP como un requisito necesario para su titulación, sino una oportunidad única de demostrar que la enseñanza universitaria es un proceso permanente de mejora continua, con innovaciones técnicas apoyado en avances científicos y tecnológicos, que les permita a los estudiantes poner en funcionamiento los conocimientos adquiridos.

La práctica profesional en el análisis y gestión de esta investigación permitió realizar la aplicación de los instrumentos de medición para las dos licenciaturas, mediante el mismo cuestionario conteniendo idénticas preguntas para ambas carreras de nivel superior. El universo de acción se circunscribe en empresas de servicios del sector público y privado, al mismo tiempo puede tener aplicación práctica en diferentes licenciaturas de otras unidades académicas de la BUAP.

\section{REFERENCIAS}

1. Álvarez González, M., \& Álvarez Justel , J. (2015). La tutoría universitaria: del modelo actual a un modelo integral. Revista Electrónica Interuniversitaria de Formación del Profesorado, 2.

2. Bravo, L. M. (1987). La investigación documental y bibliográfica. Caracas: Panapo.

3. BUAP. (02 de 12 de 2009). Universia. Obtenido de Universia: http://noticias.universia.net.mx/ vida-

4. Escamilla, M. D. (Junio de 2017). Universidad Autónoma del Estado de Hidalgo. Obtenido de Universidad Autónoma del Estado de Hidalgo: https://www.uaeh.edu.mx/docencia/VI Presentaciones/licenciatura_en_mercadotecnia/fundamentos_de_metodologia_investigacion/ PRES39.pdf

5. Gaceta Universidad BUAP. (Septiembre de 2013). Órgano Oficial de la Benemérita Universidad Autónoma de Puebla. (N. D. Peralta, Ed.) Gaceta Universidad BUAP(171), 11-24.

6. Galindo, E. M. (Agosto de 2013). Metodología de Investigación Científica. Obtenido de Metodología de Investigación Científica: http://tesis-investigacion-cientifica.blogspot.mx/2013/08/que-es-el-universo.html 
7. González González , M., \& Ramírez Ramírez , I. (2011). La formación de competencias profesionales: un reto en los proyectos curriculares universitarios. Odiseo, revista electrónica de pedagogía.

8. Hernández Sampieri, C. F. (2006). Metodología de la Investigación . McGraw-Hill Interamericana.

9. Hernández-Sampieri, R. (2020). Metodología de la investigación . España: McGraw-Hill.

10. Martínez, P., Pérez, F. J., \& Martínez, M. (2018). Aplicación de los modelos de gestión de calidad a la tutoría universitaria. Madrid, España: Revista Complutense de Educación.

11. Pérez, M. E. (2018). La Práctica Profesional Crítica, Materia Integradora en la Formación Curricular a Nivel Superior. Puebla.

12. Pérez, M. E. (2018). $\neg$ Sistemática, porque interrelaciona diversos elementos y componentes para la formación profesional. Puebla.

13. Roberto Hernández Sampieri, C. F. (1999). Metodología de la Investigación. México: McGrawHill.

14. Teddlie, C. y. (2003). Major Issues and Controversies in the Use of Mixed Methods in the Social and Behavioral Studies. Handbook of Mixed Methods in Social \& Behavioral Research. 